\section{Fatores associados à autoavaliação de saúde ruim em idosos usuários do Sistema Único de Saúde}

\author{
Factors associated with poor self-rated health \\ in elderly users of the Brazilian Unified National \\ Health System
}

\author{
${ }^{1}$ Faculdade de Enfermagem, \\ Universidade Federal de \\ Goiás, Goiânia, Brasil. \\ 2 Secretaria Municipal de \\ Saúde de Goiânia, Goiânia, \\ Brasil. \\ 3 Faculdade de Nutrição. \\ Universidade Federal de \\ Goiás, Goiânia, Brasil. \\ Correspondência \\ V. Pagotto \\ Faculdade de Enfermagem, \\ Universidade Federal de Goiás. \\ Rua 227, quadra $68 \mathrm{~s} / \mathrm{n}$, Setor \\ Leste Universitário, Goiânia \\ GO 74605-080, Brasil. \\ valeriapagotto@gmail.com
}

\section{Abstract}

This article reports on prevalence of poor selfrated health and associated factors among elderly users of the Brazilian Unified National Health System (SUS) in Goiânia, Goiás State, Brazil. The article is based on a cross-sectional study with a proportional sample of elderly in the nine health districts of Goiânia. Data were collected after a pilot study and training of field staff. The measure of association was the prevalence ratio $(P R)$ and respective confidence interval (95\%CI). Multivariate analysis was performed using hierarchical Poisson regression. In 403 elderly, prevalence of poor self-rated health was 27.5\% (95\%CI: 23.232.2). The rate was $29.7 \%$ in women and $29.1 \%$ among seniors aged 60-64 years. Variables associated with poor self-rated health according to multivariate analysis were: less than one year of schooling, lack of physical exercise, use of five or more medications, and recent weight loss. The results showed high prevalence of poor self-rated health and associated factors, including both adverse social conditions and individual health and lifestyle factors.

Aged; Self-Assessment; Drug Utilization; Weight Loss
Valeria Pagotto 1,2

Adelia Yaeko Kyosen Nakatani 1

Érika Aparecida Silveira 3

\section{Introdução}

O envelhecimento populacional, uma das maiores conquistas da humanidade, tem provocado mudanças demográficas e epidemiológicas, gerando demandas para o Sistema Único de Saúde (SUS), como aumento da utilização dos serviços de saúde, do consumo de medicamentos, bem como os custos da assistência à saúde, o que, por sua vez, exigirá a incorporação de novas tecno$\operatorname{logias} 1$.

A partir dessas demandas, torna-se necessário monitorar as condições de saúde da população a fim de possibilitar a formulação das políticas de saúde. A autoavaliação do estado de saúde é um dos indicadores recomendados pela Organização Mundial da Saúde (OMS) para avaliar a saúde das populações. É um indicador confiável e válido das condições reais e objetivas de saúde das pessoas, representando aspectos da saúde física, cognitiva e emocional 2,3.

Tem sido amplamente utilizada em pesquisas gerontológicas já que existem evidências do potencial dessa medida na predição da mortalidade e do declínio funcional independente das condições objetivas de saúde 2,4 No Brasil, a Pesquisa Nacional por Amostra de Domicílios (PNAD) é a principal fonte de informações sobre a autoavaliação de saúde ${ }^{5}$. Além da PNAD, alguns estudos foram desenvolvidos em municípios brasileiros buscando analisar a autoavalia- 
ção do estado de saúde em idosos $3,5,6,7$, sendo que a presença de doenças, número de medicamentos em uso, renda domiciliar mensal, internações, consultas médicas, dificuldade/incapacidade para atividades da vida diária, presença de sintomas depressivos e ansiosos e queixa de insônia foram os fatores mais frequentemente associados nesses estudos.

No entanto, poucos estudos buscaram compreender esse indicador em idosos usuários da Atenção Primária, o que poderá contribuir para o fortalecimento da gestão dos serviços de atenção ao idoso nesse âmbito, com foco na qualidade de vida e envelhecimento ativo.

Tendo em vista que a autoavaliação de saúde é um preditor da mortalidade e também de alterações na funcionalidade do idoso ${ }^{2}$, o objetivo deste estudo foi estimar a prevalência da autoavaliação do estado de saúde ruim e os fatores associados em idosos usuários do SUS de Goiânia, Goiás, Brasil.

\section{Método}

Este estudo está inserido na pesquisa Situação de Saúde e Indicadores Antropométricos para Avaliação do Estado Nutricional de Idosos Usuários do Sistema Único de Saúde de Goiânia - GO, também denominado Projeto Idosos/Goiânia. Tratase de um estudo de delineamento transversal, com idosos de ambos os sexos, usuários da Rede de Atenção Primária à Saúde (SUS).

Como este estudo integra o Projeto Idosos/ Goiânia, o tamanho da amostra foi definido pelo desfecho de interesse com menor prevalência. Entretanto, para verificar se a amostra do presente estudo possuía poder estatístico para identificar diferenças estatisticamente significantes, procedeu-se, a posteriori, o cálculo do tamanho da amostra. No programa Epi Info (Centers for Disease Control and Prevention, Atlanta, Estados Unidos), foram considerados os seguintes parâmetros: prevalência do desfecho autoavaliação do estado de saúde ruim/muito ruim de 13,4\%; prevalência da exposição de interesse - presença de 3 ou mais morbidades de $38 \%$ entre aqueles com autoavaliação de saúde ruim/muito ruim; nível de 95\% de confiança ( $\alpha<$ $0,05)$; poder estatístico de $80 \%$; razão de expostos: não expostos de 2:1; razão de prevalência (RP) de 2. Com esses parâmetros, encontrou-se o número de 210 , e foram acrescentados $20 \%$ para análise estratificada, mais $30 \%$ para controle de fatores de confusão e para minimizar o efeito do delineamento, obtendo-se, assim, o número de 315 idosos. Portanto, a amostra total do projeto Idosos/Goiânia foi de 418 idosos, número que contempla suficientemente os objetivos do presente estudo.

Foram incluídas pessoas com 60 anos e mais, que foram atendidas em consulta ambulatorial no período de doze meses anteriores à coleta de dados, como forma de confirmar se o idoso era usuário do SUS/Rede Primária, e que consentiram em participar do estudo mediante assinatura do Termo de Consentimento Livre e Esclarecido. Foram excluídos os idosos que apresentavam transtorno mental ou qualquer outra condição que os impossibilitasse de responder ao questionário como, por exemplo, deficiência auditiva.

O processo de amostragem foi realizado em múltiplos estágios com o objetivo de realizar seleção representativa de usuários da rede de atenção primária à saúde distribuídos nos 9 distritos sanitários (DS) do município. Assim, as seguintes etapas foram realizadas: (1) distribuição proporcional do número amostral necessário nos nove DS, conforme a população de idosos residentes; (2) identificação das unidades de maior fluxo de idosos em cada um dos DS; (3) listagem dos prontuários nas respectivas unidades de saúde dos idosos que se consultaram no último ano; (4) alocação aleatória simples dos idosos a serem convidados a participar da pesquisa por DS, conforme proporção calculada na etapa 1.

Foi realizado um estudo piloto, em área de abrangência, em uma Unidade de Atenção Primária à Saúde que não foi incluída na amostragem final. $\mathrm{O}$ estudo piloto possibilitou, além de aprimoramento da logística e dos instrumentos de coleta de dados, uma melhor estimativa do total de perdas devido à desatualização dos endereços cadastrados no SUS.

A coleta de dados foi realizada no período de novembro de 2008 a março de 2009, a partir de um questionário padronizado e pré-testado. As visitas domiciliares para a coleta foram realizadas por 9 duplas, compostas por uma antropometrista e uma entrevistadora, devidamente treinadas e padronizadas. Após o término das entrevistas, procedia-se a checagem dos questionários e codificação. Os dados foram digitados em dupla entrada, e, ao final da digitação, todas as inconsistências foram corrigidas de acordo com o questionário original.

A variável desfecho deste estudo foi a autoavaliação do estado de saúde ruim. A pergunta utilizada foi: "O que o(a) Sr(a). acha do seu estado de saúde no último mês?”. As repostas foram lidas, e o(a) idoso(a) poderia escolher uma das 5 opções de resposta: "muito bom”, "bom”, "regular", "fraco” "péssimo”. A partir disso, realizou-se a dicotomização da variável em Boa (muito bom, bom e regular) e Ruim (fraco e péssimo) 7 . 
As variáveis explanatórias foram divididas em 3 blocos: Bloco 1 - demográficas e socioeconômicas (sexo, idade, número de residentes no domicílio, viver com companheiro, cor da pele, anos de estudo e classe econômica); Bloco 2 - estilo de vida (tabagismo, consumo de bebidas alcoólicas, prática de atividade física, consumo diário de frutas e vegetais); Bloco 3 - condições de saúde (morbidades referidas, número de morbidades, perda de peso recente, estado nutricional, uso de medicamentos e hospitalização no último ano).

A variável classe econômica foi categorizada em A, B, C, D e E, conforme o Critério de Classificação Econômica da Associação Brasileira de Empresas de Pesquisa (ABEP) ${ }^{8}$. O estado nutricional foi determinado pelo índice de massa corporal (IMC), por meio de peso e altura aferidos, classificados em: baixo peso (IMC $<22 \mathrm{~kg}$ ) $\mathrm{m}^{2}$ ), normal (IMC $=22-27 \mathrm{~kg} / \mathrm{m}^{2}$ ) e obeso (IMC > $27 \mathrm{~kg} / \mathrm{m}^{2}$ ). Esses pontos de corte são específicos para a população idosa 9,10. As morbidades foram identificadas por meio das respostas à pergunta: “Quais doenças o médico já disse que o(a) $\operatorname{Sr}(a)$. têm?" e foram, posteriormente, categorizadas segundo a Classificação Internacional de Doenças - 10a Revisão (CID-10) 11.

A análise dos dados foi realizada no programa STATA, versão 9.0 (Stata Corp., College Station, Estados Unidos). Estimou-se a prevalência de autoavaliação do estado de saúde ruim para todas as variáveis do estudo, com seus respectivos intervalos de 95\% de confiança (IC95\%). Realizou-se regressão de Poisson simples para avaliar a associação entre as variáveis explanatórias e o desfecho. A associação foi estimada pela estatística Wald, considerando-se nível de 5\% de significância $(p<0,05)$. Todas as variáveis que na análise bivariada apresentaram valor $\mathrm{p} \leq 0,20$ foram inseridas no modelo de análise multivariada hierarquizada por regressão de Poisson. As variáveis foram inseridas na análise conforme os três blocos (Bloco 1, Bloco 2, Bloco 3) já citados e testados de modo distal-proximal em relação à autoavaliação do estado de saúde ruim. Mantiveram-se, no modelo multivariado de seu respectivo nível hierárquico, as variáveis que apresentaram valor $\mathrm{p} \leq 0,05$ ou quando houve mudanças na RP ajustada maiores que $10 \%$.

Este projeto de pesquisa foi aprovado pelo Comitê de Ética em Pesquisa da Universidade Federal de Goiás.

\section{Resultados}

De 418 entrevistas realizadas, 15 foram excluídas porque não tinham resposta para a pergunta so- bre a autoavaliação do estado de saúde, resultando em uma amostra final de 403 idosos.

A prevalência de autoavaliação de saúde ruim foi de 27,5\% (IC95\%: 23,2-32,2). Analisando todas as categorias da autoavaliação do estado de saúde, encontraram-se as seguintes prevalências: muito boa $3,47 \%$, boa $27,3 \%$, regular $41,6 \%$, fraca $14,14 \%$ e péssima $13,4 \%$. Na Figura 1, observa-se que a prevalência de autoavaliação de saúde ruim foi maior entre os idosos que estudaram menos de um ano (35,6\%), enquanto que a percepção de saúde muito boa/boa foi mais prevalente entre os idosos com 4-7 anos de estudo (33\%; $\mathrm{p}=0,092)$.

Observando-se as características demográficas e socioeconômicas dos idosos entrevistados, $66 \%$ eram do sexo feminino, a média de idade foi de 70 anos $( \pm 6,70)$, com predominância da faixa etária de 65-69 anos (29,8\%), 46,41\% dos idosos pertencia à classe econômica $\mathrm{C}$, seguido da classe $\mathrm{D} / \mathrm{E}(38,7 \%)$, e a média de anos de estudo foi de 3,4 anos $( \pm 3,7)$. A autoavaliação de saúde ruim foi mais prevalente no sexo feminino, na faixa etária entre 60 e 69 anos, cor da pele preta, naqueles que viviam sem companheiro, com menos de 1 ano de estudo, na classe social A/B e entre os que moravam sozinhos. Na Tabela 1, observou-se associação estatisticamente significativa com o desfecho nas categorias não viver com companheiro ( $R P=1,44$; IC95\%: 1,05-1,98) e ter menos de 1 ano de estudo (RP = 1,99; IC95\%: 1,07-3,70).

Em relação ao estilo de vida, observou-se: $9,7 \%$ fumantes, $15,9 \%$ consumidores de bebida alcoólica, $63,5 \%$ sedentários, mais de $70 \%$ não consumiam diariamente frutas e verduras. As maiores prevalências de autoavaliação do estado de saúde ruim ocorreram entre os fumantes (28,2\%), os que não consumiam bebida alcoólica (28\%), os que não praticavam atividade física $(34,8 \%)$, e entre os que não consumiam frutas (30\%) e verduras $(29,1 \%)$ diariamente. Dessas, a única variável estatisticamente associada ao desfecho foi não praticar atividade física $(\mathrm{RP}=2,32$; IC95\%: 1,52-3,53) (Tabela 2).

Quanto às condições de saúde, 43,4\% referiram ter 3 ou mais morbidades, sendo que as mais frequentes foram: hipertensão arterial $(59,8 \%)$, diabetes mellitus $(23,6 \%)$ e doenças osteomusculares (33\%). A prevalência de internações no último ano foi de $24,3 \%$; uso de medicamentos, 87,8\%; consumo de 1-2 medicamentos, $39,6 \%$. Em relação ao estado nutricional, 49,1\% apresentaram excesso de peso, e 34,9\% referiram perda de peso recente. Observou-se associação estatisticamente significante com a autoavaliação do estado de saúde ruim nas seguintes categorias: 3 ou mais morbidades ( $\mathrm{RP}=1,98$; IC95\%: 1,43-2,74), doenças osteomusculares $(\mathrm{RP}=1,78$; 
Figura 1

Prevalência da autoavaliação do estado de saúde de idosos usuários do SUS, conforme anos de estudo. Goiânia, Goiás, Brasil, $2009(p=0,092)$.

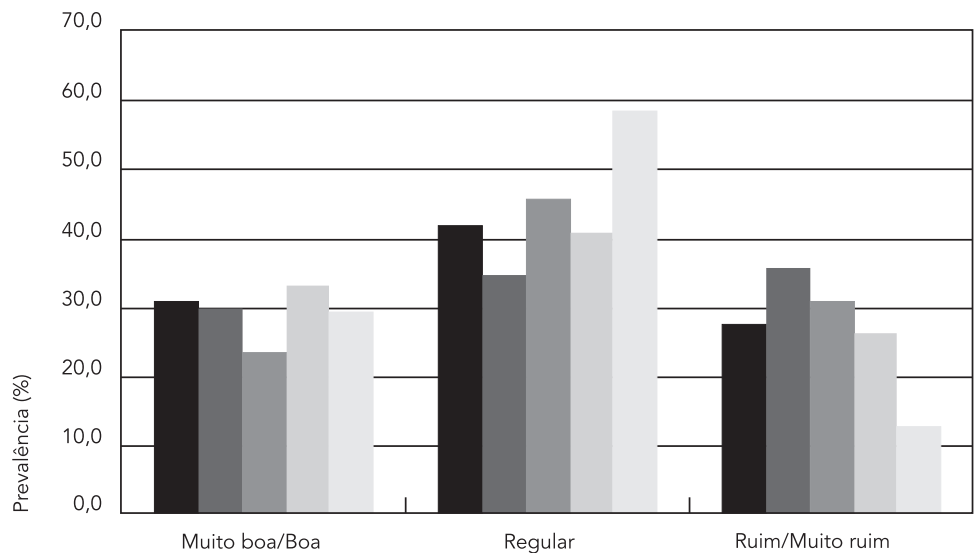

Muito boa/Boa
Regular

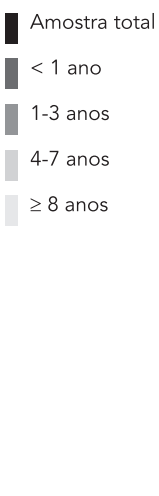

Autoavaliação do estado de saúde

Tabela 1

Distribuição dos idosos, prevalência e fatores associados à autoavaliação do estado de saúde ruim segundo variáveis demográficas e socioeconômicas em idosos usuários do SUS. Goiânia, Goiás, Brasil, 2009 (n = 403).

\begin{tabular}{|c|c|c|c|c|c|c|}
\hline \multirow[t]{2}{*}{ Variáveis } & \multirow[t]{2}{*}{$\mathbf{n}$} & \multirow[t]{2}{*}{$\%$} & \multicolumn{2}{|c|}{$\begin{array}{l}\text { Prevalência da } \\
\text { autoavaliação } \\
\text { de saúde ruim }\end{array}$} & \multirow[t]{2}{*}{ RP (IC95\%) } & \multirow[t]{2}{*}{ Valor de $p$ * } \\
\hline & & & $\mathbf{n}$ & $\%$ & & \\
\hline Sexo & & & & & & 0,186 \\
\hline Feminino & 266 & 66,0 & 79 & 29,7 & $1,27(0,89-1,81)$ & \\
\hline Masculino & 137 & 34,0 & 32 & 23,4 & 1,00 & \\
\hline Faixa etária (anos) & & & & & & 0,957 \\
\hline $60-64$ & 110 & 27,3 & 32 & 29,1 & $1,12(0,74-1,71)$ & \\
\hline $65-69$ & 120 & 29,8 & 31 & 25,8 & 1,00 & \\
\hline $70-74$ & 86 & 21,3 & 24 & 27,9 & $1,08(0,68-1,70)$ & \\
\hline$\geq 75$ & 87 & 21,6 & 24 & 27,6 & $1,06(0,67-1,68)$ & \\
\hline Cor da pele & & & & & & 0,684 \\
\hline Branca & 186 & 46,2 & 51 & 27,4 & $1,04(0,74-1,47)$ & \\
\hline Parda & 171 & 42,4 & 45 & 26,3 & 1,00 & \\
\hline Preta & 46 & 11,4 & 15 & 32,6 & $1,24(0,76-2,01)$ & \\
\hline Vive com companheiro & & & & & & 0,022 \\
\hline Sim & 226 & 56,1 & 52 & 23,0 & 1,00 & \\
\hline Não & 177 & 43,9 & 59 & 33,3 & $1,44(1,05-1,98)$ & \\
\hline Anos de estudo & & & & & & $0,092 * \star$ \\
\hline$<1$ & 104 & 28,8 & 37 & 35,6 & $1,99(1,07-3,70)$ & \\
\hline $1-3$ & 94 & 26,0 & 29 & 30,8 & $1,73(0,91-3,27)$ & \\
\hline $4-7$ & 115 & 31,9 & 30 & 26,1 & $1,36(0,71-2,62)$ & \\
\hline$\geq 8$ & 48 & 13,3 & 6 & 12,5 & 1,00 & \\
\hline
\end{tabular}

(continua) 
Tabela 1 (continuação)

\begin{tabular}{|c|c|c|c|c|c|c|}
\hline \multirow[t]{2}{*}{ Variáveis } & \multirow[t]{2}{*}{$\mathrm{n}$} & \multirow[t]{2}{*}{$\%$} & \multicolumn{2}{|c|}{$\begin{array}{l}\text { Prevalência da } \\
\text { autoavaliação } \\
\text { de saúde ruim }\end{array}$} & \multirow[t]{2}{*}{ RP (IC95\%) } & \multirow[t]{2}{*}{ Valor de $p$ * } \\
\hline & & & $\mathrm{n}$ & $\%$ & & \\
\hline Classe social & & & & & & 0,731 \\
\hline$A / B$ & 60 & 14,9 & 19 & 31,7 & $1,18(0,76-1,84)$ & \\
\hline C & 187 & 46,4 & 50 & 26,7 & 1,00 & \\
\hline $\mathrm{D} / \mathrm{E}$ & 156 & 38,7 & 42 & 26,9 & $1,01(0,71-1,43)$ & \\
\hline Residentes no domicílio & & & & & & 0,725 \\
\hline Mora sozinho & 38 & 9,4 & 13 & 34,2 & $1,36(0,80-2,30)$ & \\
\hline 2 pessoas & 139 & 34,6 & 35 & 25,2 & 1,00 & \\
\hline $3-4$ pessoas & 158 & 39,3 & 44 & 27,8 & $1,10(0,75-1,61)$ & \\
\hline 5 ou mais & 67 & 16,7 & 19 & 28,4 & $1,13(0,70-1,81)$ & \\
\hline
\end{tabular}

IC95\%: intervalo de 95\% de confiança; RP: razão de prevalência.

* Teste de Wald;

** Qui-quadrado para tendência linear.

Tabela 2

Prevalência de autoavaliação do estado de saúde ruim e fatores associados segundo variáveis de estilo de vida em idosos usuários do SUS. Goiânia, Goiás, Brasil, 2009 ( $n=403)$.

\begin{tabular}{|c|c|c|c|c|c|c|}
\hline \multirow[t]{2}{*}{ Variáveis } & \multirow[t]{2}{*}{$\mathrm{n}$} & \multirow[t]{2}{*}{$\%$} & \multicolumn{2}{|c|}{$\begin{array}{l}\text { Prevalência de } \\
\text { autoavaliação } \\
\text { de saúde ruim }\end{array}$} & \multirow[t]{2}{*}{ RP (IC95\%) } & \multirow[t]{2}{*}{ Valor de $p$} \\
\hline & & & $n$ & $\%$ & & \\
\hline Tabagismo & & & & & & 0,996 \\
\hline Fumante & 39 & 9,7 & 11 & 28,2 & $1,02(0,59-1,78)$ & \\
\hline Ex-fumante & 175 & 43,4 & 48 & 27,4 & $1,01(0,71-1,39)$ & \\
\hline Não fumante & 189 & 46,9 & 52 & 27,5 & 1,00 & \\
\hline Consumo de bebida alcoólica & & & & & & 0,668 \\
\hline Sim & 64 & 15,9 & 16 & 25,0 & 1,00 & \\
\hline Não & 339 & 84,1 & 95 & 28,0 & $1,12(0,71-1,77)$ & \\
\hline Atividade física & & & & & & 0,000 \\
\hline Sim & 147 & 36,5 & 22 & 15,0 & 1,00 & \\
\hline Não & 256 & 63,5 & 89 & 34,8 & $2,32(1,52-3,53)$ & \\
\hline Consumo diário de frutas & & & & & & 0,090 \\
\hline Sim & 108 & 26,9 & 22 & 20,4 & 1,00 & \\
\hline Não & 293 & 73,1 & 88 & 30,0 & $1,47(0,97-2,22)$ & \\
\hline Consumo diário de verduras & & & & & & 0,302 \\
\hline Sim & 116 & 28,9 & 27 & 23,3 & 1,00 & \\
\hline Não & 285 & 71,1 & 83 & 29,1 & $1,25(0,85-1,82)$ & \\
\hline
\end{tabular}

IC95\%: intervalo de 95\% de confiança; RP: razão de prevalência.

* Teste de Wald. 
IC95\%: 1,31-2,43), diabetes mellitus $(\mathrm{RP}=1,57$; IC95\%: 1,05-2,34), hospitalização no último ano $(\mathrm{RP}=1,68$; IC95\%: 1,23-2,31), uso de 5 ou mais medicamentos (RP = 1,70; IC95\%: 1,15-2,49) e perda de peso recente $(\mathrm{RP}=1,46$; IC95\%: 1,05 2,02) (Tabela 3).

As variáveis que entraram na análise multivariada foram: Bloco 1 - sexo, viver com companheiro e anos de estudo; Bloco 2 - prática de atividade física e consumo diário de frutas; Bloco 3 - número de morbidades, diabetes mellitus, doenças osteomusculares, hospitalização no último ano, uso de medicamentos e perda de peso recente.
Após análise multivariada (Tabela 4), conforme modelo de análise hierarquizado, as variáveis que permaneceram associadas à autoavaliação de saúde ruim foram: escolaridade, atividade física, medicamentos em uso e perda de peso. Dessas variáveis, pela interpretação do IC95\%, as categorias associadas ao desfecho foram: menos de 1 ano de estudo ( $R P=1,99$; IC95\%: 1,07-3,70), não praticar atividade física $(\mathrm{RP}=2,17$; IC95\%: $1,44-3,66)$, uso de 5 ou mais medicamentos ( $\mathrm{RP}=$ 1,68; IC95\%: 1,10-2,56), perda de peso no último ano (RP = 1,52; IC95\%: 1,06-2,15).

\section{Tabela 3}

Distribuição da amostra, prevalência e fatores associados à autoavaliação do estado de saúde ruim segundo variáveis de condições de saúde em usuários do SUS. Goiânia, Goiás, Brasil, 2009 ( $n=403)$.

\begin{tabular}{|c|c|c|c|c|c|c|}
\hline \multirow[t]{2}{*}{ Variáveis } & \multirow[t]{2}{*}{$\mathbf{n}$} & \multirow[t]{2}{*}{$\%$} & \multicolumn{2}{|c|}{$\begin{array}{l}\text { Prevalência de } \\
\text { autoavaliação } \\
\text { de saúde ruim }\end{array}$} & \multirow[t]{2}{*}{ RP (IC95\%) } & \multirow[t]{2}{*}{ Valor de $p$ * } \\
\hline & & & n & $\%$ & & \\
\hline Número de morbidades & & & & & & 0,000 \\
\hline $0-2$ & 228 & 56,6 & 44 & 19,3 & 1,00 & \\
\hline 3 ou mais & 175 & 43,42 & 67 & 38,3 & $1,98(1,43-2,74)$ & \\
\hline \multicolumn{7}{|l|}{ Morbidades referidas } \\
\hline Hipertensão arterial & 241 & 59,8 & 70 & 29,0 & $1,17(0,83-1,63)$ & 0,359 \\
\hline Diabetes mellitus & 95 & 23,6 & 36 & 37,9 & $1,57(1,05-2,34)$ & 0,006 \\
\hline Doenças osteomusculares & 133 & 33,0 & 52 & 46,8 & $1,78(1,31-2,43)$ & 0,000 \\
\hline Doenças digestivas & 48 & 18,8 & 30 & 63,8 & $1,04(0,79-1,37)$ & 0,770 \\
\hline Doenças cardiovasculares & 62 & 15,4 & 23 & 34,3 & $1,28(0,86-1,89)$ & 0,316 \\
\hline Doenças respiratórias & 42 & 16,0 & 52 & 39,1 & $1,03(0,92-1,16)$ & 0,543 \\
\hline Internação no último ano & & & & & & 0,001 \\
\hline Sim & 98 & 24,3 & 39 & 39,8 & $1,68(1,23-2,31)$ & \\
\hline Não & 305 & 75,7 & 72 & 23,6 & 1,00 & \\
\hline Uso de medicação & & & & & & 0,619 \\
\hline Sim & 354 & 87,8 & 99 & 28,0 & $1,14(0,68-1,92)$ & \\
\hline Não & 49 & 12,2 & 12 & 24,5 & 1,00 & \\
\hline Medicamentos em uso & & & & & & $0,010 * \star$ \\
\hline $1-2$ & 140 & 39,7 & 32 & 22,9 & 1,00 & \\
\hline $3-4$ & 102 & 28,9 & 24 & 23,5 & $1,03(0,65-1,64)$ & \\
\hline 5 ou mais & 111 & 31,4 & 43 & 38,7 & $1,70(1,15-2,49)$ & \\
\hline Estado nutricional & & & & & & 0,399 \\
\hline Baixo peso & 62 & 15,4 & 20 & 32,3 & $1,35(0,85-2,16)$ & \\
\hline Eutrófico & 143 & 35,5 & 34 & 23,8 & 1,00 & \\
\hline Excesso de peso & 198 & 49,1 & 57 & 28,8 & $1,21(0,84-1,75)$ & \\
\hline Perda de peso & & & & & & 0,023 \\
\hline Sim & 137 & 34,9 & 46 & 33,6 & $1,46(1,05-2,02)$ & \\
\hline Não & 256 & 65,1 & 59 & 23,0 & 1,00 & \\
\hline
\end{tabular}

IC95\%: intervalo de 95\% de confiança; RP: razão de prevalência.

* Teste de Wald;

** Qui-quadrado para tendência linear. 
Razão de prevalência (RP) ajustada hierarquicamente para prevalência de autoavaliação do estado de saúde ruim em idosos usuários do SUS. Goiânia, Goiás, Brasil, 2009 ( $n=403)$.

\begin{tabular}{|c|c|c|c|}
\hline Variáveis & RP ajustada & IC95\% & Valor de $p$ \\
\hline \multicolumn{4}{|l|}{ 1으 nível } \\
\hline Anos de estudo & & & 0,009 \\
\hline$<1$ & 1,99 & $1,07-3,70$ & \\
\hline $1-3$ & 1,73 & $0,91-3,27$ & \\
\hline $4-7$ & 1,36 & $0,71-2,62$ & \\
\hline$\geq 8$ & 1,00 & & \\
\hline \multicolumn{4}{|l|}{ 2oo nível } \\
\hline Atividade física & & & 0,000 \\
\hline Sim & 1,00 & & \\
\hline Não & 2,17 & $1,44-3,66$ & \\
\hline \multicolumn{4}{|l|}{ 3o nível } \\
\hline Medicamentos em uso & & & 0,000 \\
\hline $1-2$ & 1,00 & & \\
\hline $3-4$ & 0,99 & $0,60-1,62$ & \\
\hline 5 ou mais & 1,68 & $1,10-2,56$ & \\
\hline Perda de peso & & & 0,021 \\
\hline Sim & 1,52 & $1,06-2,15$ & \\
\hline Não & 1,00 & & \\
\hline
\end{tabular}

IC95\%: intervalo de 95\% de confiança.

\section{Discussão}

Este é o primeiro estudo a analisar a autoavaliação do estado de saúde em idosos no Município de Goiânia e na Região Centro-oeste. Os resultados desta investigação ampliam o conhecimento sobre a saúde dos idosos nesse município e no Brasil. Dessa forma, podem contribuir para as atividades de gestão no SUS, ensino, pesquisa, além de direcionar as ações de promoção e atenção à saúde para a população idosa no país.

A prevalência de autoavaliação do estado de saúde ruim de 27,5\% (IC95\%: 23,2-32,2) mostrouse semelhante às observadas em outros estudos desenvolvidos em países como Japão (26,9\%) 12 e Cingapura (27,6\%) 13. Por outro lado, foi superior à encontrada no Texas, Estados Unidos (22,6\%) 14 e na Espanha (13,6\%) 15 e inferior à encontrada em Cuba, no Chile e no México (entre 60\%-70\%) 16.

No Brasil, resultado semelhante foi encontrado no Projeto Bambuí 7 . Estudo realizado em 15 capitais brasileiras 17 demonstrou que, entre as pessoas de 50 anos ou mais, a prevalência de percepção de saúde regular/ruim foi maior em São Luís (Maranhão) e Natal (Rio Grande do Norte), e a menor prevalência foi encontrada em Porto Alegre (Rio Grande do Sul) 18. A prevalência de autoavaliação do estado de saúde ruim difere entre as regiões brasileiras, com valores mais elevados na Região Nordeste. Souza et al. 17 apontam que há uma homogeneidade interna dos percentuais encontrados nas regiões Norte/Nordeste e Sul/Sudeste e uma heterogeneidade entre essas regiões, demonstrando que as diferenças regionais refletem as desigualdades sociais e de saúde no país.

As condições socioeconômicas têm importância marcante nos estudos sobre autoavaliação de saúde, pois refletem o cenário de desigualdades sociais no Brasil e suas repercussões para a saúde da população ${ }^{5}$. No presente estudo, não houve associação entre classe econômica e a autoavaliação de saúde ruim, o que talvez se deva à alta proporção de idosos nas classes econômicas $\mathrm{C}$ e D/E $(85,1 \%)$, refletindo as baixas condições econômicas dos idosos usuários do SUS.

Assim como em outras pesquisas, observouse associação entre autoavaliação de saúde ruim e anos de estudo, menos de um ano. Pesquisas apontam que a autoavaliação do estado de saúde melhora à medida que aumenta o nível de informação das pessoas 7,17,19. Os autores citados afirmam que a educação determina diversas vantagens para a saúde. Um melhor nível educacional poderia favorecer o acesso às informações, a modificação do estilo de vida, a adoção 
de hábitos saudáveis, a procura pelos serviços de saúde, o ingresso em atividades que priorizam a promoção da saúde e o seguimento correto das orientações relacionadas à obtenção de melhor qualidade de vida 17,19 .

Quanto ao sexo, não foram observadas diferenças significantes, porém, estudos que analisaram a população sem discriminar entre usuários do SUS demonstraram que as mulheres avaliam sua saúde de forma pior comparadas aos homens 14,15.

Embora não tenha ocorrido associação com a idade, observou-se que, na faixa etária mais avançada (75 anos ou mais), houve uma prevalência maior de autoavaliação de saúde boa, quando comparada à faixa etária mais jovem. Resultado similar foi encontrado por Damian et al. 15 e parece ser resultante de um processo de adaptação dos idosos ou do reconhecimento da deterioração das condições de saúde como normal do envelhecimento. Também, pode estar relacionado à sobrevivência, que seleciona, com o avançar da idade, os idosos mais saudáveis. Esse resultado vem confirmar as evidências de que os idosos mais velhos são, atualmente, um grupo bastante distinto, tornando-se essencial investigar mais criteriosamente esses indivíduos.

A presença de doenças é um dos fatores relevantes nas pesquisas sobre autoavaliação do estado de saúde, apresentando associações significativas com a percepção ruim 7,17,20. Quanto ao tipo de morbidade referida, alguns autores encontraram elevada proporção de autoavaliação de saúde ruim entre os idosos com diabetes $3,13,14,20$ e com doenças osteomusculares 14 . Neste estudo, não houve associação entre autoavaliação de saúde ruim e o tipo de morbidade, bem como com o número total de doenças. Estudos indicam que idosos com até três doenças crônicas podem se considerar saudáveis quando as mesmas estão controladas 15,19. Além disso, a percepção de ser doente pode estar mais relacionada às incapacidades do que a ser portador de doenças crônicas 19. Estudo qualitativo que buscou compreender os significados atribuídos à autoavaliação da saúde do idoso revelou que a ideia mais presente na compreensão de saúde pelos idosos está vinculada a permanecer ativo dentro das capacidades físicas e de mobilidade, executar os próprios desígnios e ser independente nas atividades cotidianas 6 .

Após a análise multivariada, não se observou associação entre hospitalização e autoavaliação de saúde ruim, resultado diferente do encontrado em outros estudos 7,21. Isso pode estar relacionado ao tipo de informação levantada na presente pesquisa, pois foi questionado ao idoso sobre as hospitalizações no último ano. Como a questão sobre autoavaliação de saúde se referia ao momento presente, hospitalizações no passado podem não ter influenciado a sua percepção de saúde atual.

A polifarmácia, uso de 5 ou mais medicamentos, permaneceu associada à autoavaliação do estado de saúde ruim após análise multivariada, corroborando achados de outros estudos 7,13. As múltiplas doenças nos idosos levam ao consumo de múltiplos medicamentos. Embora os medicamentos contribuam para o controle das doenças crônicas, o tratamento medicamentoso geralmente é prolongado, fato que aumenta o risco de reações adversas, interações medicamentosas e falhas no cumprimento do tratamento 22 . Além disso, a falta de autonomia de muitos idosos, o baixo nível de escolaridade e de atenção com muitos medicamentos e ainda o pouco esclarecimento oferecido com relação ao tratamento podem contribuir com confusões e uso inadequado dos medicamentos, que, por sua vez, colaboram com reações adversas e interações e que, por fim, contribuem para uma percepção negativa da saúde 6,22.

A perda de peso também se associou significativamente à autoavaliação do estado de saúde ruim, conforme resultados de outras pesquisas 7,12. Os idosos apresentam condições peculiares que condicionam o seu estado nutricional às alterações fisiológicas próprias do envelhecimento, às enfermidades presentes, aos fatores socioeconômicos, psicológicos e de estrutura familiar ${ }^{23}$. A presença de múltiplas doenças e as hospitalizações frequentes, além do baixo nível socioeconômico, perda de autonomia e solidão contribuem para a perda de peso do idoso 21,23 . Esses fatores contribuintes para a perda de peso também podem estar relacionados à percepção negativa de saúde, uma vez que estão inseridos em todo um contexto negativo. A perda de peso é uma variável importante que exige vigilância e cuidados de qualquer equipe de profissionais da saúde, pois implica em riscos de outras morbidades e até mortalidade. Além disso, é um dos fatores que determinam o início do ciclo da fragilidade entre os idosos 24 .

Não praticar atividade física apresentou-se como o mais importante fator associado à autoavaliação do estado de saúde ruim, confirmando achados de outros estudos 7,14,13. Almeida et al. 25 apontam que os fatores que podem contribuir para a não participação de idosos em atividades físicas e prática de esporte são a dificuldade de locomoção, a falta de entusiasmo, a ausência de estímulo interno e externo, o estilo de vida, a falta de oferta de atividades culturais com práticas corporais e ainda a falta de condições socioeconômicas. Em função da cau- 
salidade reversa própria de estudos transversais, essa associação deve ser analisada com cautela, pois não se pode afirmar que o idoso não pratica atividade física porque está com percepção da saúde ruim ou, ao contrário, por sentir sua saúde ruim, parou de praticar atividade física.

A prevalência elevada de autoavaliação do estado de saúde ruim confirma resultados de outros estudos. No entanto, observou-se que os fatores associados a uma percepção ruim de saúde estão relacionados aos hábitos de vida e saúde (praticar atividade física, uso medicamentos, perda de peso) e a questões sociais (anos de estudo) do que propriamente à presença de doenças.

Dessa forma, os achados deste estudo reforçam a hipótese de que múltiplas questões estão envolvidas na forma com que as pessoas idosas avaliam a sua saúde, compreendendo diversas dimensões da vida e não apenas a presença ou ausência de doenças. É importante que os serviços de saúde do SUS, ao traçarem suas estratégias de ações, tenham atenção e vigilância especial para os idosos que possuem essas características: baixo nível de escolaridade, que fazem uso de múltiplos medicamentos, que não praticam atividade física e que apresentem perda peso, tendo em vista que esses fatores estão associados à percepção ruim da saúde e são relacionados a desfechos clínicos que comprometem a qualidade de vida do idoso, podendo, inclusive, contribuir para a síndrome de fragilidade e risco de mortalidade 2,24 .

Tendo em vista que se trata de uma amostra de idosos usuários da Atenção Primária/SUS, esses dados contribuem para a formulação de políticas públicas e para redirecionar a atenção à saúde da pessoa idosa no país. A promoção de estilos de vida mais saudáveis, com a participação da Atenção Primária e seus profissionais, pode representar um grande impacto na melhoria dos indicadores de saúde do idoso e nos custos relacionados à gestão dos serviços. Ações integradas que contemplem todas essas dimensões podem contribuir para o bem-estar do idoso e consequentemente para sua qualidade de vida.

\section{Resumo}

Foram investigados a prevalência e os fatores associados à autoavaliação do estado de saúde ruim em idosos usuários do SUS, em Goiânia, Goiás, Brasil. Estudo transversal, com amostra de idosos proporcional aos nove Distritos Sanitários de Goiânia. Os dados foram coletados após estudo piloto e treinamento da equipe de campo. A medida de associação foi a razão de prevalência $(R P)$ e respectivos intervalos de $95 \%$ de confiança (IC95\%). Realizou-se análise multivariada por regressão de Poisson hierarquizada. Em 403 idosos, a prevalência de autoavaliação do estado de saúde ruim foi de 27,5\% (IC95\%: 23,2-32,2), sendo 29,7\% nas mulheres e 29,1\% entre idosos com 60-64 anos de idade. As variáveis associadas após análise multivariada foram: ter menos de 1 ano de estudo, não praticar atividade física, uso de 5 ou mais medicamentos e perda de peso recente. Os resultados demonstraram elevada prevalência de autoavaliação de saúde ruim, e os fatores associados compreendem desde as condições sociais do idoso até as suas condições de saúde e estilo de vida.

Idoso; Auto-Avaliação; Uso de Medicamentos; Perda de Peso

\section{Colaboradores}

Todos os autores tiveram igual participação: concepção do projeto, análise e interpretação dos dados; redação do artigo e revisão crítica relevante do conteúdo intelectual; aprovação final da versão a ser publicada.

\section{Agradecimentos}

Ao Conselho Nacional de Desenvolvimento Científico e Tecnológico (CNPq, edital universal 014/2008) pelo financiamento da pesquisa. 


\section{Referências}

1. Lima-Costa MF, Loyola Filho AI, Matos DL. Tendências nas condições de saúde e uso de serviços de saúde entre idosos brasileiros: um estudo baseado na Pesquisa Nacional por Amostra de Domicílios (1998, 2003). Cad Saúde Pública 2007; 23:2467-78.

2. Idler EL, Benyamini Y. Self-Rated Health and Mortality: a review of twenty-seven community studies. J Health Soc Behav 1997; 38:21-37.

3. Theme Filha MM, Szwarcwald CL, Souza Júnior PRB. Socio-demographic characteristics, treatment coverage, and self-rated health of individuals who reported six chronic diseases in Brazil, 2003. Cad Saúde Pública 2005; 21 Suppl 1:S43-53.

4. DeSalvo KB, Bloser MHAN, Reynolds K, He J, Muntner P. Mortality preditction with a single general self-rated health question. A meta-analysis. J Gen Intern Med 2005; 21:267-75.

5. Dachs JNW, Santos APRS. Autoavaliação do estado de saúde no Brasil: análise dos dados da PNAD/2003. Ciênc Saúde Coletiva 2006; 11:887-94.

6. Fonseca MGUP, Firmo JOA, Loyola-Filho AI, Uchoa E. Papel da autonomia na autoavaliação da saúde do idoso. Rev Saúde Pública 2010; 44:159-65.

7. Lima-Costa MF, Firmo JOA, Uchoa EA. A estrutura da autoavaliação da saúde entre idosos: projeto Bambuí. Rev Saúde Pública 2004; 38:827-34.

8. Associação Brasileira das Empresas de Pesquisa. Critério de Classificação Econômica Brasil. 2008. http://www.abep.org/novo/Utils/FileGenerate. ashx?id=12 (acessado em Mar/2009).

9. Lipschtz D. A. screening for nutritional status in the elderly. Prim Care 1994; 21:55-67.

10. Silveira EA, Kac G, Barbosa LS. Prevalência e fatores associados à obesidade em idosos residentes em Pelotas, Rio Grande do Sul, Brasil: classificação da obesidade segundo dois pontos de corte do índice de massa corporal. Cad Saúde Pública 2009; 25:1569-77.

11. Organização Mundial da Saúde. Classificação estatística internacional de doenças e problemas relacionados à saúde. 10a Revisão. São Paulo: Centro Colaborador da Organização Mundial da Saúde/ Editora da Universidade de São Paulo; 2007.

12. Sun W, Watanabe M, Tanimoto Y, Shibutani T, Kono R, Saito M, et al. Factors associated with good selfrated health of non-disabled elderly living alone in Japan: a cross-sectional study. BMC Public Health 2007; 7:297-306.

13. Ramkumar A, Quah JLS, Wong T, Yeo LS, Nieh CC, Shankar A, et al. Self-rated health, associated factors and diseases: community-based cross-sectional study of Singaporean adults aged 40 years and above. Ann Acad Med Singapore 2009; 38:606-12.
14. Phillips LJ, Hammock RL, Blanton JM. Predictors of self-rated health status among Texas residents. Rural Remote Health 2005; 2:1-10.

15. Damian J, Ruigomez A, Pastor V, Martin-Moreno JM. Determinants of self-assessed health among Spanish older people living at home. J Epidemiol Community Health 1999; 53:412-6.

16. Wong R, Peláez M, Palloni A. Autoinforme de salud general en adultos mayores de América Latina y el Caribe: su utilidad como indicador. Rev Panam Salud Pública 2005; 17:323-32.

17. Souza MC, Otero UB, Almeida LM, Turci SRB, Figueiredo VC, Lozana JA. Autoavaliação de saúde e limitações físicas decorrentes de problemas de saúde. Rev Saúde Pública 2008; 42:741-9.

18. Paskulin LMG, Vianna LAC. Perfil sociodemográfico e condições de saúde autorreferidas de idosos de Porto Alegre. Rev Saúde Pública 2007; 41:757-68.

19. Alves LCA, Rodrigues RN. Determinantes da autopercepção de saúde entre idosos do Município de São Paulo, Brasil. Rev Panam Salud Pública 2005; 17:333-41.

20. Lima MG, Barros MBA, César CLG, Goldbaum M, Carandina L, Ciconelli RM. Impacto of chronic disease on quality of life among the elderly in the state of São Paulo, Brazil: a population-based study. Rev Panam Salud Pública 2009; 25:314-21.

21. Siqueira AB, Cordeiro RC, Perracini MR, Ramos LR. Impacto funcional da internação hospitalar de pacientes idosos. Rev Saúde Pública 2004; 38:687-94.

22. Medeiros-Souza P, Santos-Neto LL, Kusano LTE, Pereira MG. Diagnosis and control of polypharmacy in the elderly. Rev Saúde Pública 2007; 41: 1049-53.

23. Campos MAG, Pedroso ERP, Lamounier JA, Colosimo EA, Abrantes MM. Estado nutricional e fatores associados em idosos. Rev Assoc Med Bras (1992) 2006; 52:214-21.

24. Fried LP, Tangen CM, Walston J, Newman AB, Hirsch C, Gottdiener J, et al. Frailty in older adults: evidence for a phenotype. J Gerontol A Biol Sci Med Sci 2001; 56:146-56.

25. Almeida MMG, Moreira RF, Araújo TM, Pinho OS. Atividades de lazer entre idosos, Feira de Santana, Bahia. Rev Saúde Pública 2005; 29:339-52.

Recebido em 30/Set/2010

Versão final reapresentada em 27/Abr/2011

Aprovado em 06/Mai/2011 